\title{
Free-surface pressure distributions with minimum wave resistance
}

\author{
E. O. Tuck L. Lazauskas*
}

(Received 25 January 2001)

\begin{abstract}
The wave resistance of distributions of excess pressure over a rectangular region on the surface of a steady stream is minimised by choice of spatial variation in pressure. Both unconstrained and constrained (non-negative) pressures are studied. Results with impressive resistance reductions are provided, both via discretisation to a large number of step pressures, and via optimisation within a low-order continuous family.
\end{abstract}

*Department of Applied Mathematics, The University of Adelaide, Australia 5005. mailto: etuck@maths.adelaide.edu.au

${ }^{0}$ See http://anziamj.austms.org.au/V43/E026 for this article and ancillary services, (C) Austral. Mathematical Soc. 2001. Published 9 October 2001. 


\section{Contents}

1 Introduction $\quad$ E76

2 The wave resistance integral $\quad$ E78

3 Constant-pressure patches $\quad$ E80

4 Rectangular patches $\quad$ E81

5 Unconstrained optima $\quad$ E82

6 Constrained optima $\quad$ E89

7 Near-optimal continuous pressures $\quad$ E93

8 Conclusion $\quad$ E97

$\begin{array}{lr}\text { References } & \text { E99 }\end{array}$

\section{Introduction}

Hovercraft $[13,6]$ travelling over water are supported by fans whose main role is to exert a downward pressure on the water surface. If we ignore the detail of the actual air flow, a given hovercraft may be considered to be 
specified by a prescribed excess pressure on the water relative to the ambient atmospheric pressure. This excess pressure need not be (and in general is not) uniform over the planform of the hovercraft, and the question posed here relates to choices of spatial pressure variations that minimise wavemaking when the hovercraft moves steadily forward on a calm sea. There are other potential applications of travelling pressure distributions, e.g. to travelling meteorological disturbances, or even to conventional surface vessels, where the pressure distribution models the hydrodynamic forces on the vessel's hull.

We actually seek to minimise the wave resistance, which is a measure of the total energy in the wave pattern behind the travelling disturbance, and we do so subject to a fixed total vertical force exerted by the fans, which in the hovercraft application must balance its weight. The wave resistance is a quadratic functional of the pressure distribution, so that this optimisation problem is in principle a straightforward exercise in the calculus of variations [2], and leads to a linear integral equation to determine the optimum pressure. We use a step-function discretisation to convert the continuous optimisation problem to a quadratic algebraic optimisation problem, which in the absence of constraints requires only solution of linear algebraic equations.

However, in general the solution of this problem requires pressures that can take some negative values, and this may not be acceptable in the application context. Hence it is also necessary to consider a constrained problem where the pressures are required to be non-negative. The discretised problem is then a standard quadratic programming [10] problem, and again solutions can be found easily. 
Results are given for rectangular disturbance regions, for a range of speeds. The fundamental character of the discretised optimal solutions, both unconstrained and constrained, varies dramatically but smoothly with speed. We also conjecture properties of some near-optimal continuous solutions.

Theoretical and computational attempts have been made to reduce or eliminate the waves made by ships and other travelling free-surface disturbances for as long as there have been theories, and for example the many papers of Havelock [7] include such studies. For a review including somewhat more recent studies on minimisation of ship wave resistance, see Wehausen [15, p. 205]. For travelling pressure disturbances, important early works on minimisation of wave resistance include those of Bessho [1] and Maruo [8], and more recently Doctors [4] (see also Doctors and Day [5]) has optimised unconstrained families of pressures, involving up to 4 distinct "subcushion" parameters. The present work can be considered a constrained extension of [4] to many (e.g. 100) parameters, with indications of convergence toward a continuous optimum.

\section{The wave resistance integral}

The wave resistance or $\operatorname{drag} R_{W}$ of a pressure distribution $p(x, y)$ acting on a region $S$ of the $(x, y)$ plane while moving forward with speed $U$ is [16, p. 598]

$$
R_{W}=\frac{\kappa^{2}}{2 \pi \rho U^{2}} \int_{-\pi / 2}^{\pi / 2} \sec ^{5} \theta|\Omega(\theta)|^{2} d \theta
$$


where

$$
\Omega(\theta)=\iint_{S} p(x, y) e^{i \kappa x \sec \theta+i \kappa y \sec ^{2} \theta \sin \theta} d x d y .
$$

Here $\rho$ is the water density, $g$ the acceleration of gravity, and $\kappa=g / U^{2}$. The water depth has been taken to be infinite, and the water is inviscid and incompressible. The pressure $p(x, y)$ has been assumed small enough to generate only small disturbances to a free surface that is initially plane. The quantity $\Omega(\theta)$ is proportional to the amplitude of the component of the wave pattern made by the moving pressure that is propagating at a direction making an angle $\theta$ to the direction of forward motion, and the wave resistance is a measure of the total energy in this wave pattern.

An equivalent formula is

$$
R_{W}=\frac{\kappa^{2}}{4 \rho U^{2}} \iint_{S} d x d y p(x, y) \iint_{S} d \xi d \eta p(\xi, \eta) W(\kappa(x-\xi), \kappa(y-\eta))
$$

where

$$
W(X, Y)=\frac{2}{\pi} \int_{-\pi / 2}^{\pi / 2} \sec ^{5} \theta \cos (X \sec \theta) \cos \left(Y \sec ^{2} \theta \sin \theta\right) d \theta .
$$

Equation (3) thus defines $R_{W}$ to be a positive definite quadratic functional in $p(x, y)$, with a symmetric kernel function $W(X, Y)$.

The net lift force exerted by this pressure distribution is

$$
L=\iint_{S} d x d y p(x, y)
$$


and we are interested in minimising the drag $R_{W}$ within families of pressure distributions $p(x, y)$ having fixed values of the lift $L$. In the absence of further constraints, this optimisation can proceed directly as in the calculus of variations [2], which shows that the optimal pressure is proportional to a solution of the first-kind integral equation

$$
\iint_{S} d \xi d \eta p(\xi, \eta) W(\kappa(x-\xi), \kappa(y-\eta))=1
$$

However, we proceed indirectly, by first discretising the drag formula.

\section{Constant-pressure patches}

Consider a region $S$ of the $(x, y)$ plane that is subdivided into $N$ patches $S_{j}$, $j=1,2, \ldots, N$, on each of which the pressure is assumed to be constant, namely $p=p_{j}$. Then (3) states that

$$
\rho U^{2} \kappa^{2} R_{W}=\sum_{j=1}^{N} \sum_{k=1}^{N} A_{j k} p_{j} p_{k}
$$

where

$$
A_{j k}=\frac{1}{4} \kappa^{4} \iint_{S_{j}} d x d y \iint_{S_{k}} d \xi d \eta W(\kappa(x-\xi), \kappa(y-\eta))
$$


The drag has now become an algebraic quadratic form in the $N$-vector $\left\{p_{j}\right\}$, with symmetric matrix $A_{j k}$. Similarly the lift force is then

$$
L=\sum_{j=1}^{N} p_{j} s_{j}
$$

where $s_{j}$ is the area of the $j$ th patch.

The (unconstrained) problem of drag minimisation within a family of pressures with equal lift $L$ has thus reduced to a quadratic algebraic optimisation problem, and hence $p_{j}$ is proportional to solutions of the linear equations

$$
\sum_{k=1}^{N} A_{j k} p_{k}=s_{j}
$$

\section{Rectangular patches}

The above applies for individual patches of any shape and location. Suppose now for simplicity that the patches are all rectangles, and that the $j$ th patch $S_{j}$ has centre $\left(\bar{x}_{j}, \bar{y}_{j}\right), x$-wise length $2 a_{j}$, and $y$-wise breadth $2 b_{j}$. Then it follows directly from (8) and (4), or (somewhat more easily) indirectly from (1) and (2), that

$$
A_{j k}=\frac{16}{\pi} \int_{0}^{\pi / 2} \frac{\cos \theta}{\sin ^{2} \theta} \sin \alpha_{j} \sin \alpha_{k} \sin \beta_{j} \sin \beta_{k} \cos \left(\bar{\alpha}_{j}-\bar{\alpha}_{k}\right) \cos \left(\bar{\beta}_{j}-\bar{\beta}_{k}\right) d \theta
$$


where

$$
\begin{aligned}
& \alpha_{j}=\kappa a_{j} \sec \theta, \quad \beta_{j}=\kappa b_{j} \sec ^{2} \theta \sin \theta, \\
& \bar{\alpha}_{j}=\kappa \bar{x}_{j} \sec \theta \quad \text { and } \quad \bar{\beta}_{j}=\kappa \bar{y}_{j} \sec ^{2} \theta \sin \theta .
\end{aligned}
$$

The non-dimensional matrix coefficients $A_{j k}$ can be evaluated by direct numerical quadrature, e.g. using Simpson's rule. The integrand in (11) oscillates rapidly as $\theta \rightarrow \pi / 2$, and many integrand evaluations are required, at least 50,000 for 3-figure accuracy.

For $N=1$ (i.e. a single rectangular constant-pressure patch) equation (7) reduces to $\rho U^{2} \kappa^{2} R_{W}=A_{11} p_{1}^{2}$. In the general case of a region with area $S$, we define a mean pressure $p_{0}=L / S$, and a drag coefficient

$$
C_{D}=\frac{\rho g}{2 b p_{0}^{2}} R_{W}
$$

Then for a single rectangular patch for which $p_{0}=p_{1}$, we have $2 \kappa b C_{D}=A_{11}$. This formula and computations from it agree with Newman and Poole [9] and Doctors and Sharma [3]. More generally $2 \kappa b C_{D}$ can be identified with the series on the right of (7) providing the pressures $p_{j}$ have been normalised by dividing by their mean $p_{0}$, which we assume to be the case.

\section{Unconstrained optima}

The linear algebraic equations (10) can be solved by any convenient code. This can be done for any region $S$ inside which $N$ rectangular patches can 
be placed. These patches need not fill $S$, nor need they form a uniform grid.

Nevertheless, it is convenient as our example region in the present paper to take $S$ to be itself a rectangle of length $2 a$ and width $2 b$, with a uniform rectangular grid which in the first instance is assumed to fill $S$ completely. A typical $20 \times 20$ grid used in our computations has $N=400$, with all patches having $a_{j}=a / 20, b_{j}=b / 20$, and uniformly spaced centres. However, as it can be shown that the optimum pressure distribution possesses both $x$-wise and $y$-wise symmetry, the number of distinct unknowns $p_{j}$ can be reduced to the 100 in each quadrant of the rectangle.

We have for the present purpose standardised on $b / a=0.5$, which is typical of a hovercraft, and have particularly emphasised the case $\kappa a=1$ corresponding to a length-based Froude number of $F=U / \sqrt{2 a g} \approx 0.71$. At this Froude number, a single constant-pressure patch has near-maximum drag, namely $C_{D}=2.265$. On the other hand, our optimised pressure distribution with 400 patches has $C_{D}=0.259$ at $F=0.71$, a 9-times reduction!

Although on the face of it, this very low drag is a very promising improvement relative to uniform pressure, the actual pressure distribution shown in Figure 3 tells a somewhat less satisfactory story. The price paid for substantial drag reduction is wild swings between very large positive and negative pressures. This pressure distribution is quite unrealistic, and is unlikely to be achievable in practice for hovercraft, or in other potential applications. Similar conclusions were arrived at by Doctors and Day [5].

Nevertheless, these results are correctly optimal and exact (within the 
3-figure accuracy of our computations) for the assumed stepwise-constant pressure distribution. Some distributions with much lower $N$, while not so impressive in drag reduction, would be feasible in applications if variations of pressure consisting of a small number of steps were allowed. For example, the results for a uniform $4 \times 4$ grid prescribe 4 distinct positive pressures (namely $\left.p_{j}=0.047,0.390,1.510,2.053\right)$, and yield $C_{D}=1.633$. The general feature of these low $-N$ results (at least for $b / a=0.5$ and $\kappa a=1$ ) is for the pressures to be high near each end and low in the middle. Doctors and Day [5] have also studied some low-order step-like pressure distributions, and have noted that at some speeds negative pressures are a feature of the optima even when there are only a small number of steps.

As we increase $N$, some of the more-central pressures take negative values, which can be considered infeasible, or at least undesirable in applications. An obvious expedient then is to eliminate some patches that potentially could yield negative pressures, so less-than-completely filling the bounding rectangular region with patches of positive pressure. Although some satisfactory trial-and-error results were obtained this way, a systematic quadratic programming approach is preferred.

Figures $1-5$ also show the pressure distributions producing minimum drag at some other Froude numbers, i.e. other values of $\kappa a$, and indicate that the qualitative features of the optimal pressure distribution vary systematically with Froude number, or effectively with speed.

At low speeds, the main characteristic of the pressure distribution is the 


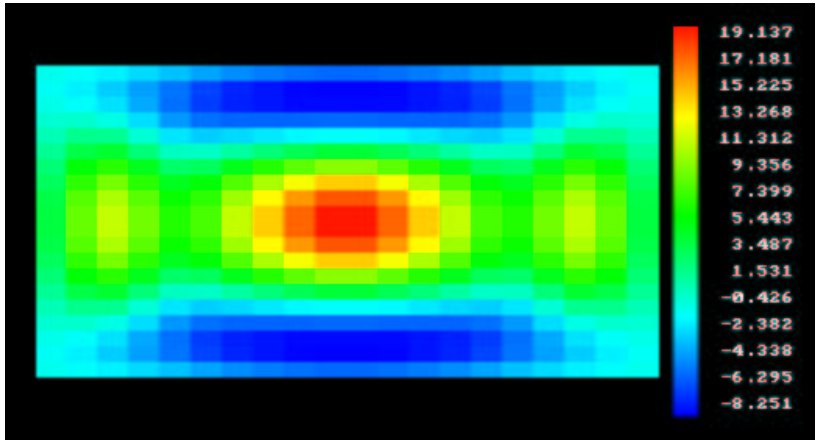

FiguRE 1: Unconstrained optimal step-function pressure distributions for $20 \times 20$ grid, at $b / a=0.5$ and $F=0.40$.

existence of a strip of positive pressure that runs down the centreline of the vessel, and is bounded on either side by thin longitudinal strips of negative pressure. For $F=0.4$ (Figure 1), the positive pressure tends to be concentrated near the centre of the vessel, and is of scaled magnitude about $p=+19$.

As the Froude number is increased to about $F=0.47$ (Figure 2), the positive pressure strip running down the centreline increases in length and the maximum pressure increases to $p=+24$. The two negative strips become shorter in length and of greater magnitude, with minima $p=-19$.

As $F$ is increased further to $F=0.71$ (Figure 3), the central positive pres- 


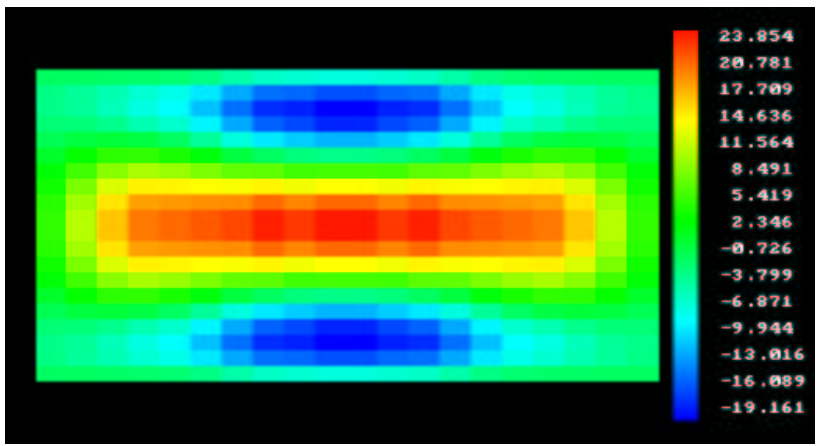

FIGURE 2: Unconstrained optimal step-function pressure distributions for $20 \times 20$ grid, at $b / a=0.5$ and $F=0.47$.

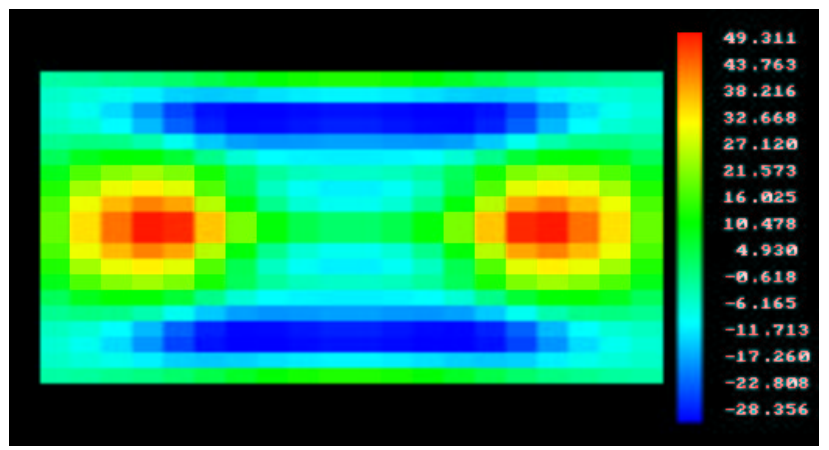

FIGURE 3: Unconstrained optimal step-function pressure distributions for $20 \times 20$ grid, at $b / a=0.5$ and $F=0.71$. 
sure strip breaks up into two separate sharp positive pressure peaks located at about $20 \%$ of the length from the ends of the vessel, and separated by a region of relatively small-magnitude negative pressure. The maximum pressure of the positive peaks is now extremely high at about $p=+49$. At the same time, the negative pressure strips near the sides of the vessel lengthen again, and their minimum pressure decreases further to about $p=-28$. This is the case $\kappa a=1$ discussed above.

Between $F=0.71$ and $F=1.0$, the region of negative pressure at the centre of the vessel deepens and becomes more significant, while the negative strips at the sides of the vessel become shorter and less significant. At about $F=0.75$ the negative-pressure region forms an "I" shape, and at higher $F$ the side strips disappear altogether. The two regions of large positive pressure remain situated at about $20 \%$ of the length from the ends of the vessel, each occupying quite a small area. At $F=1$ (Figure 4), the maximum pressure is about $p=+48$, while the minimum pressure has dropped to $p=-50$ and is located at the centre of the vessel.

Beyond $F=1$, the two positive-pressure regions move closer to the ends of the vessel, until they become concentrated essentially in two transverse strips at bow and stern. The pressure in these strips varies roughly parabolically across the full width of the rectangle, reaching a maximum at the centreline of about $p=+28$ at $F=1.5$ (Figure 5). At the same time, the region of negative pressure increases in extent, occupying most of the central portion of the vessel. As the region of negative pressure increases in size, its absolute magnitude decreases, the minimum pressure reaching $p=-8$ at $F=1.5$. 


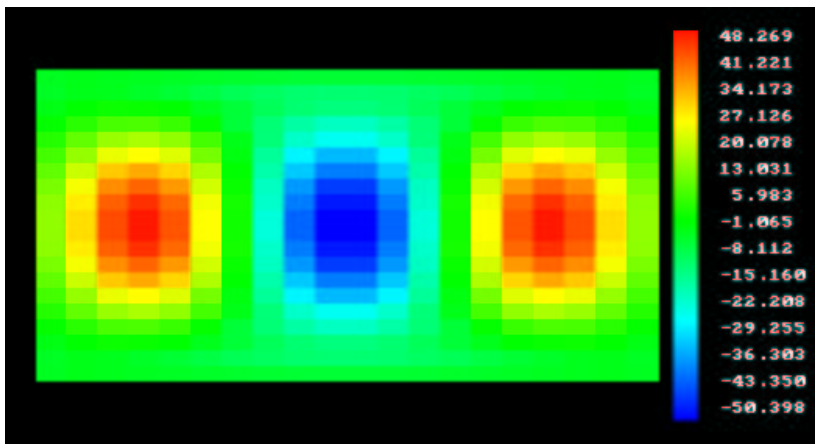

FiguRE 4: Unconstrained optimal step-function pressure distributions for $20 \times 20$ grid, at $b / a=0.5$ and $F=1.00$.

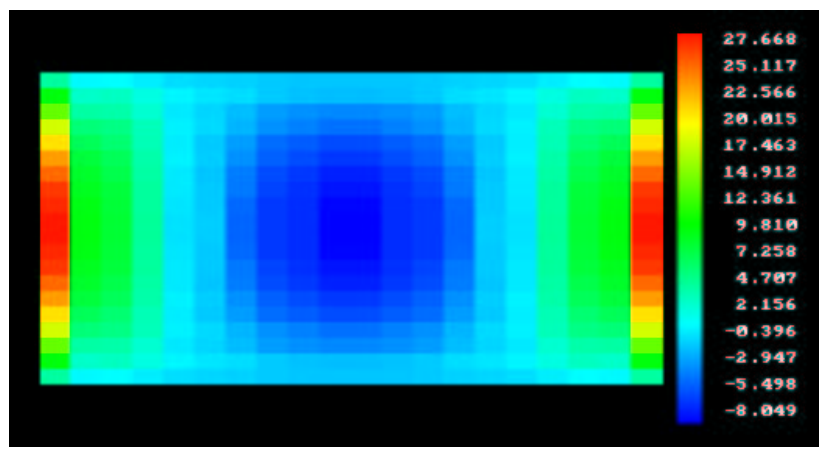

FIGURE 5: Unconstrained optimal step-function pressure distributions for $20 \times 20$ grid, at $b / a=0.5$ and $F=1.50$. 
A plot versus Froude number of the lowest wave resistance coefficient $C_{D}$ that is achievable on this $20 \times 20$ grid is given as the dotted curve in Figure 11 .

\section{Constrained optima}

Since negative pressures can be considered infeasible in applications of the present optimisation, we must add the non-negativity constraint $p(x, y) \geq 0$ to the continuous optimisation problem, or

$$
p_{j} \geq 0
$$

to the discretised version.

The problem of minimising the algebraic quadratic form (7) subject to the equality constraint (9) and the inequality constraint (14) is a standard one in quadratic programming [10], and there are efficient algorithms for its solution. In effect these algorithms systematically decide which patches to eliminate from the optimisation process by setting their pressure to zero.

Figures $6-10$ show the resulting optimal non-negative pressure distribution on the $20 \times 20$ grid for $b / a=0.5$, at five selected speeds. For example, at $\kappa a=1$ or $F \approx 0.71$ (Figure 8 ), the drag is now $C_{D}=0.999$. Although this is nowhere near as low as was achieved without constraints, it is still an impressive (better than half) reduction relative to the constant-pressure baseline $C_{D}=2.265$. 


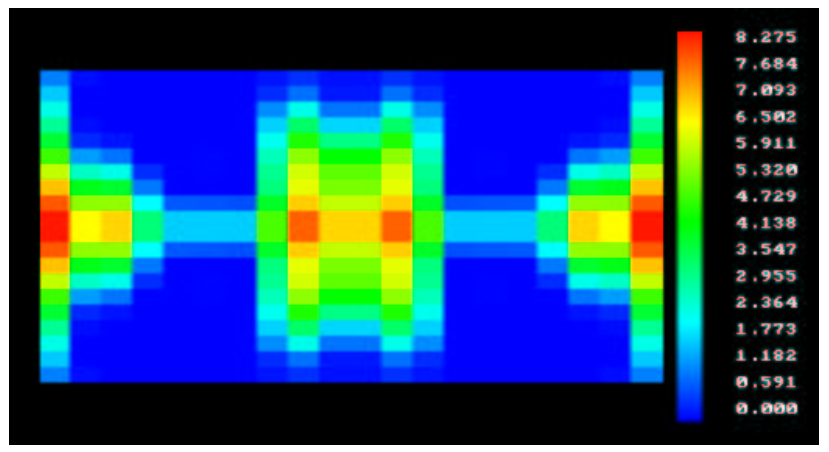

FIGURE 6: Constrained (non-negative) optimal step-function pressure distributions for $20 \times 20$ grid, at $b / a=0.5$ and $F=0.40$.

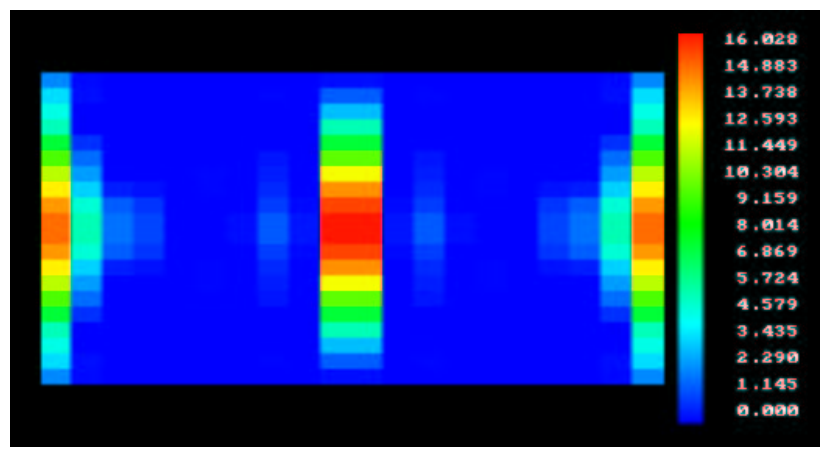

FiguRE 7: Constrained (non-negative) optimal step-function pressure distributions for $20 \times 20$ grid, at $b / a=0.5$ and $F=0.47$. 


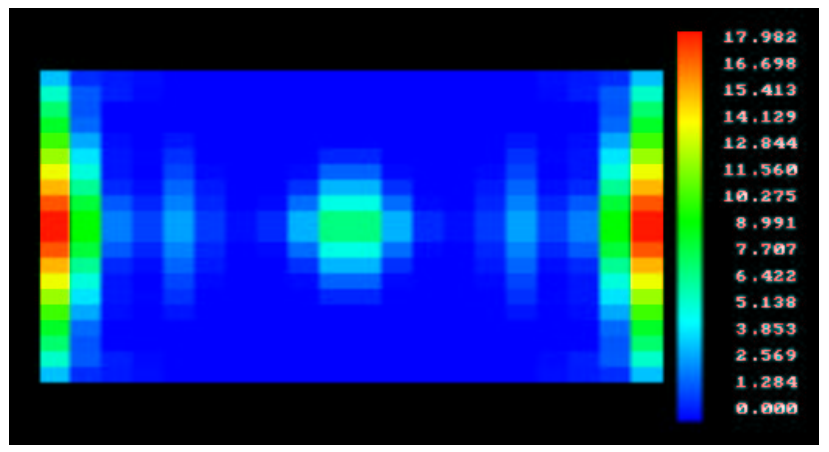

FIGURE 8: Constrained (non-negative) optimal step-function pressure distributions for $20 \times 20$ grid, at $b / a=0.5$ and $F=0.71$.

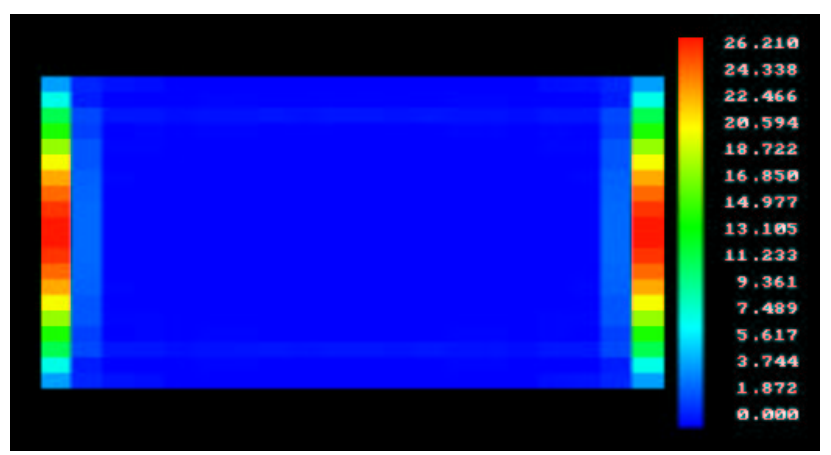

Figure 9: Constrained (non-negative) optimal step-function pressure distributions for $20 \times 20$ grid, at $b / a=0.5$ and $F=1.00$. 


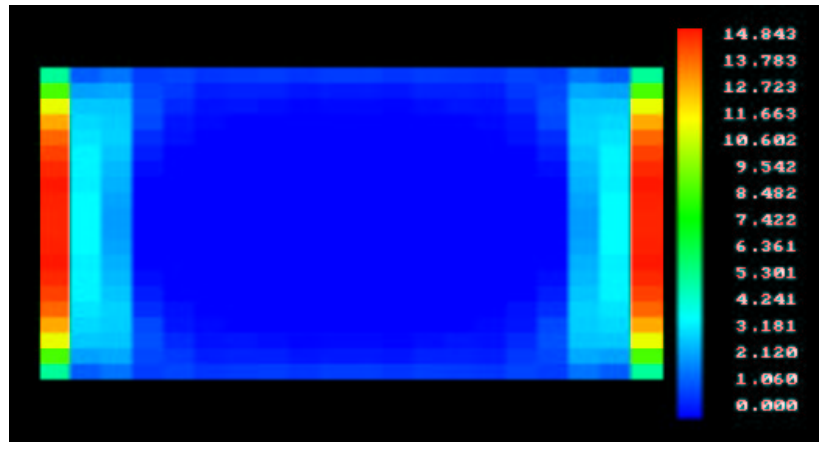

FiguRE 10: Constrained (non-negative) optimal step-function pressure distributions for $20 \times 20$ grid, at $b / a=0.5$ and $F=1.50$.

As with the unconstrained pressure case, there is a smooth variation in the optimal pressure distribution as the speed is varied. The most noticeable feature of the optimal pressures at all of the speeds shown in Figures 6-10 is dominant peaks at the bow and stern. At low speeds there is also a significant central peak. The actual magnitudes of the positive maxima are much less than those for equivalent unconstrained optima, as is to be expected, and this is another benefit of the constraint. Although there are other complex features to the distribution, these involve only very small pressures relative to these main three peaks, a characteristic that we shall exploit in the following section.

A plot versus Froude number of the wave resistance coefficient $C_{D}$ for 
these optimal constrained pressures is given as the dashed curve in Figure 11. Although this is indeed the best result that can be achieved with non-negative pressure on a $20 \times 20$ grid of constant-pressure elements, it is not quite as low as can be achieved with some continuous non-negative pressure distributions, as we shall see below.

\section{Near-optimal continuous pressures}

The structure of the constrained discretised results obtained above suggests an attempt to minimise wave resistance within a family of continuous pressure distributions involving three separate positive pressure patches, one at each end and one in the centre of the rectangle. The end pressures should extend across the whole width of the rectangle, and decay toward zero as we approach the sides of the rectangle, but the central pressures need not extend to the whole width of the rectangle. At most speeds, a parabolic smooth $y$-variation was found by experimentation to perform well, whereas little benefit was found from use of anything other than uniform $x$-wise pressure within each separate patch.

Hence we consider the following pressure family.

$$
p(x, y)= \begin{cases}p_{1}\left[1-(y /(\sigma b))^{2}\right], & |x|<\epsilon_{1} a,|y|<\sigma b \\ p_{2}\left[1-(y / b)^{2}\right], & \left(1-\epsilon_{2}\right) a<|x|<a \\ 0 & \text { otherwise }\end{cases}
$$


where $p_{1}$ and $p_{2}$ are positive constants chosen so that the mean pressure over the whole rectangle is normalised to unity as usual. Thus only the ratio $p_{1} / p_{2}$ is significant, and we choose to measure this in terms of the fraction $\phi$ of the total lift that is borne by the central patch. This family thus has 4 independent parameters $\phi, \sigma, \epsilon_{1}$ and $\epsilon_{2}$. The central patch has dimensions $2 \epsilon_{1} a$ by $2 \sigma b$, i.e. $\epsilon_{1}$ measures its longitudinal and $\sigma$ its lateral fractional extent. The parameter $\epsilon_{2}$ measures the longitudinal fractional extent of the two end patches, which each have dimensions $\epsilon_{2} a$ by $2 b$.

We now write down from (1) and (2) the wave resistance $R_{W}$ for this family, and minimise it with respect to these 4 parameters. In fact, for all speeds of interest here, our conclusion is that $\epsilon_{2}=0$ is best, so the optimum longitudinal extent of the end patches is zero, even though they bear finite load! That is, the best end patches are of large aspect ratio, with large pressures to compensate for their small chord, and may be called "pressure lines" by analogy with lifting lines in aerodynamics.

Meanwhile, the relative importance and dimensions of the central patch change with speed in a systematic smooth manner. There are essentially three speed ranges at $b / a=0.5$.

For $F>0.95$, there should be no central patch at all, i.e. we find $\phi=0$. (Actually $\phi<0$ would perform even better at some speeds in this range, but this would violate the non-negativity constraint.) The optimal pressure distribution (within the present family) is thus simply two pressure lines in tandem, located at bow and stern. The optimal design then has some 
similarity with a vessel supported by forward and aft hydrofoils, each of large aspect ratio.

For $0.65<F<0.95$, there should also be a central pressure line, i.e. we find $\phi>0$ but $\epsilon_{1}=0$. The values of $\phi$ and $\sigma$ vary with speed in this range, which includes the speed with maximum wave resistance.

Finally, for $0.40<F<0.65$, the optimal central patch has $\epsilon_{1}>0$, i.e. is of finite aspect ratio. At the low end of this range, the central patch has become dominant, with $\phi \approx 1$, and extends across the whole width, with $\sigma=1$. If $F<0.40$, so long as $p \rightarrow 0$ at the sides of the rectangle, the wave resistance is so small that further reduction in its magnitude is of little consequence.

Figure 11 displays our final results as a function of Froude number, for $b / a=0.5$. At each speed, the lowest achievable wave resistance coefficient $C_{D}$ within this family is plotted in graph (a), and the character of the optimal pressure distribution indicated via plots of $\sigma$ in graph (b) and $\phi$ in graph (c). Over a large speed range, this "optimum" has less than half of the corresponding wave resistance [9] for a constant-pressure patch. Even lower wave resistances are possible within extended families, but we believe that the present family already does very well. For example, the optimum discretised wave resistance for the rectangular $20 \times 20$ grid (shown as the dashed curve in Figure 11) is at all speeds a little higher than that shown in the solid curve.

An example suggesting even lower wave resistance is that at very high speeds, an elliptic variation in pressure with respect to $y$ is guaranteed to 

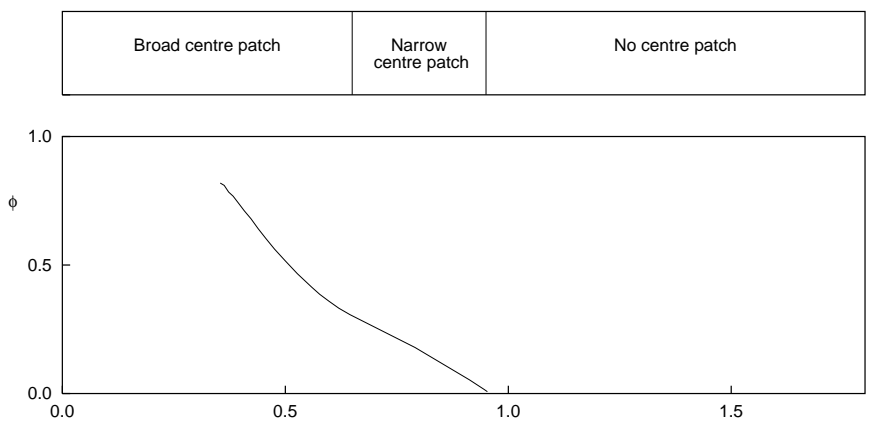

Figure

11:

(a) Drag coefficient $C_{D}$ versus Froude number at $b / a=0.5$ for near-optimum

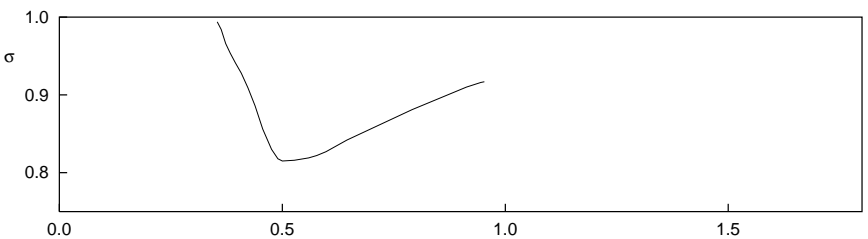
continuous family (solid curve). (b) Lateral fractional extent $\sigma$ of centre patch.

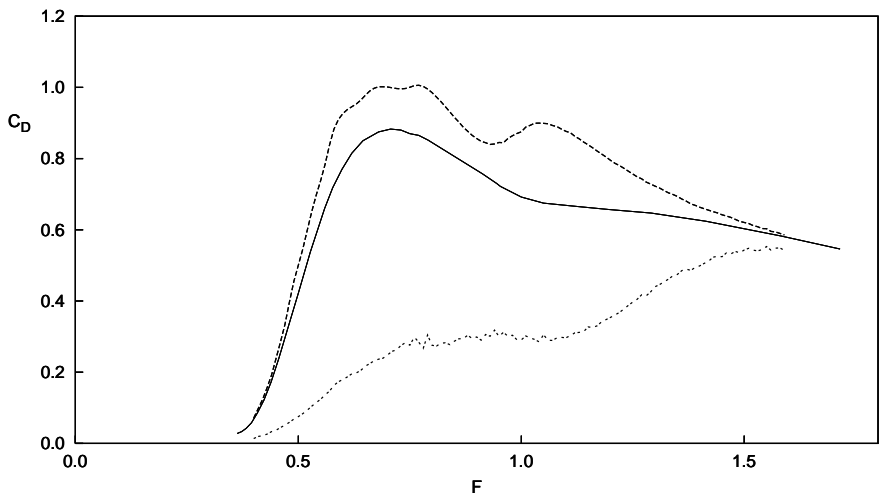

(c) Fraction $\phi$ of total load borne by centre patch. Plot (a) also shows minimised drag for a $20 \times 20$ discretisation, constrained (dashed curve) and unconstrained (dotted curve). 
be optimal. This is because in this limit, the wave resistance is given by a formula identical to that for the induced drag of a lifting surface, and the well-known Munk optimal elliptic-loading result of aerodynamics (see Thwaites [12, p. 303]) applies. In fact, we find that elliptic loading is only preferable to parabolic loading for $F>1.35$, and then only by at most about $10 \%$. There is no theoretical reason why parabolic loading should be optimal at any speed, but it does seem to perform better than some other $y$-variations tried, at least in the range $0.40<F<1.35$.

\section{Conclusion}

Near-optimum positive pressure distributions on a rectangular region with aspect ratio 0.5 are well behaved and vary smoothly with respect to speed, while reducing the wave resistance to less than about a half of that for a uniform pressure patch. Allowing negative pressures would permit even lower wave resistance (as indicated by the dotted curve in Figure 11), but with a number of disadvantages, not only that of the infeasibility of negative pressures in applications. There is a tendency for the unconstrained optimum pressures to be large and rapidly varying spatially and with respect to speed, a tendency that is reduced by the non-negativity constraint.

In the present paper we have approached the optimum in two ways, first by a systematic but still somewhat coarse 100-unknown step-function discretisation, and secondly by optimising within a special family of continuous 
pressure distributions. We have confidence that the true constrained optimum is close to what each of these methods suggests.

Some results have also been obtained at other aspect ratios $b / a$. An important feature of the constrained optimisation in the speed range studied here is that the optimum positive pressures extend to the corners of the rectangle, i.e. that the hovercraft "tries" to be as long and as wide as it can. We would therefore expect that (with the length held fixed) wider rectangles would permit lower wave resistance. This is a "high" Froude number conclusion, since it corresponds to the usual aerodynamic preference for wings of high aspect ratio, and appears to hold over the whole Froude number range $F>0.4$ of interest here. We expect however that in a more ship-like range with lower Froude numbers and lower $b / a$ values, this trend must reverse and confirm the usual naval architectural preference for slenderness.

The present paper has emphasised just one output from the hydrodynamics, namely the wave resistance. Another important output is the actual free-surface deformation produced by the pressure distribution, both in the far field where it generates the wave resistance and is potentially environmentally harmful, and in the near field close to or under the pressure patch. The former is considerably easier to compute, and Doctors and Day [5] have computed far-field waves for their low-order optimum pressures. On the other hand, the latter is of particular importance in the inverse or design problem for planing vessels, where the pressure distribution is that created by a given moving hull of small draft. In a separate study [11] (see also [14]), we have developed efficient numerical methods for generating this free-surface output, 
both in the near and far field.

Acknowledgement: This work was supported by the Australian Research Council.

\section{References}

[1] Bessho, M., On the problem of the minimum wave-making resistance of ships, Memoirs of the Defence Academy of Japan 2 (1962) 1-30. E78

[2] Craggs, J.W., Calculus of Variations, George Allen and Unwin, 1973. E77, E80

[3] Doctors, L.J. and Sharma, S.D., The wave resistance of an air-cushion vehicle in steady and accelerated motion, J. Ship Res. (1972) 248-260. E82

[4] Doctors, L. J., Optimal pressure distributions for river-based air-cushion vehicles, Schiffstechnik 44 (1997) 32-36. E78

[5] Doctors, L.J. and Day, A.H., Wave-free river-based air-cushion vehicles. Proc. Int. Conf. Hydrodynamics of High-Speed Craft Wake Wash and Motions Control, London, November 2000. E78, E83, E84, E98 
[6] Everest, J.T. and Hogben, N., Research on hovercraft over calm water, Trans RINA 109 (1967) 311. E76

[7] Havelock, T.H., Collected papers, ed. C. Wigley, Office of Naval Research, Washington D.C., 1963. E78

[8] Maruo, H., (in Japanese) J. Soc. Nav. Archs. Japan 81 (1949). E78

[9] Newman, J.N. and Poole, F.A.P., The wave resistance of a moving pressure distribution in a canal, Schiffstechnik 9 (1962) 1-6. E82, E95

[10] More, J.J. and Wright, S.J., Optimization Software Guide, SIAM, Philadelphia 1993. E77, E89

[11] Scullen, D.C. and Tuck, E.O., Free-surface elevation due to moving pressure distributions in three dimensions, in preparation, January 2001. E98

[12] Thwaites, B., Incompressible Aerodynamics, Oxford University Press, 1960. E97

[13] Trillo, R. L., Marine Hovercraft Technology, Leonard Hill Publishers, London 1971. E76

[14] Tuck, E.O., Scullen, D.C. and Lazauskas, L., Ship-wave patterns in the spirit of Michell, IUTAM Conference on Free Surface Flows, Birmingham, July 2000. Proc. ed. Y.Shikhmurzaev, The University of Birmingham, 2001. E98 
[15] Wehausen, J.H., The wave resistance of ships, Advances in Applied Mechanics, 13 (1973) 93-245. E78

[16] Wehausen, J.H. and Laitone, E.H., Surface waves, in Handbuch der Physik, ed. Flügge S., Ch. 9. Springer-Verlag, 1962. E78 